\title{
Persistently Elevated Troponin LeVels With a Negative Cardiac WorkUp: Now What?
}

\section{Pamela Cines, $M D$}

\section{Case Presentation}

A 44 year-old female with a past medical history significant only for iron deficiency anemia and syncope presented to an outside hospital with abdominal pain. The patient initially noted the pain while shopping; she described it a sharp sensation, regionalized to the right-epigastric region. When the pain recurred with greater intensity an hour later now with resulting nausea and right-arm radiation, she proceeded to her nearest ED.

Her medical history was significant for iron deficiency anemia, which with she was diagnosed during pregnancy. She had a recent hospitalization for syncope and reported positive cardiac enzymes, however she had a normal cardiac stress test. Surgical history was significant for an uncomplicated caesarian section. She reports having no allergies to medicines and her only medications are a daily aspirin and ibuprofen as needed. Social history was notable only for a five pack-year history of smoking; she quit approximately five years prior to admission.

At the OSH, physical examination was reported to be unremarkable. Her laboratory data showed a hemoglobin level of $9 \mathrm{~g} / \mathrm{dL}$, a normal chemistry panel and a troponin of $1.46 \mathrm{ng} / \mathrm{mL}$. EKG demonstrated normal sinus rhythm without any ischemic changes. CT scan and ultrasound of her abdomen were performed and were unrevealing. The patient was transferred to TJUH for further evaluation of her troponin elevation.

On admission to TJUH, the patient was afebrile with a heart rate of 58, a blood pressure of $96 / 31 \mathrm{mmHg}$ without evidence of orthostasis, respirations of 16 , and oxygen saturation of $98 \%$ on room air. She was oriented and in no distress. Physical exam was pertinent for clear lungs on pulmonary auscultation; no jugular venous distension, pedal edema, diminished pulses, or extra heart sounds on cardiovascular examination; minimal tenderness in the right upper quadrant, a well-healed caesarean section scar, but otherwise a benign abdominal examination. Hemoccult examination of the stool was negative. The EKG performed on admission to TJUH again demonstrated normal sinus rhythm, heart rate 55, without evidence of ST-T wave abnormalities.

Laboratory evaluation again showed a microcytic anemia with an iron saturation of $4 \%$ and a troponin of $1.48 \mathrm{ng} / \mathrm{mL}$. Transthoracic echocardiogram demonstrated an ejection fraction of $65 \%$ with no contraction abnormalities or evidence of valvular disease. Cardiac catheterization was performed revealing no evidence of occlusive coronary artery disease. Gastroenterology consult was obtained for evaluation of her abdominal pain. The patient underwent a right upper quadrant ultrasound and upper endoscopy, both of which were normal.

The patient remained symptom-free throughout her three-day hospitalization at TJUH, she was discharged to home in stable condition. Curiously, her troponin level on day of discharge remained elevated at $1.48 \mathrm{ng} / \mathrm{mL}$.

\section{Discussion}

Cardiac troponins are regulatory proteins controlling the interaction between actin and myosin mediated by calcium. In most normal subjects, cardiac troponins are completely undetectable. Troponin levels will begin to rise in 4 to 6 hours after an acute insult to cardiac tissue-usually related to lack of blood flow-and will remain elevated for up to 10 days, permitting late diagnosis.

In Western Medicine, and especially in Emergency Medicine, any level of detectable troponin is typically regarded as the sine qua non of an acute coronary syndrome. Indeed, according to the ACC/ESC consensus document, "any amount of myocardial damage implies an impaired clinical outcome for the patient" and that there is "no discernible threshold below which an elevated value of cardiac troponin would be deemed harmless. The ACC/AHA guidelines do indicate that myocardial necrosis as reflected by elevated troponin levels may not be due to coronary artery disease; and thus a diagnosis of myocardial ischemia, or infarction, should be supported by additional clinical and electrocardiographic evidence.

While myocardial ischemia may imply elevated troponin levels, the converse is not always true. Disease states such as sepsis, atrial fibrillation, CHF exacerbation, pulmonary embolism, myocarditis, myocardial contusion, and renal failure are also associated with detectable troponin.

In 2002 Bakshi et al enrolled 21 consecutive patients with elevated troponin who had either normal coronary angiography or stenosis of less than $50 \%$ without complex features or thrombus. Among the enrollees troponin elevations ranged from 0.21 to 54.79. Following unrevealing angiography, patients were classified as to the likely cause of their elevated troponin. Eleven of the 21 patients had tachycardia, strenuous exercise, $\mathrm{CHF}$, and pericarditis ascribed to the cause of their elevated troponins. For the remaining 10 patients, however, no clear precipitating event could be identified.

More importantly, a study by $\mathrm{Ng}$ et al reviewed 1000 consecutive patients presenting to a large, suburban hospital ED. One hundred and twelve of these patients had elevated troponin levels, but amazingly, $45 \%$ of them had a final diagnosis other than acute coronary syndrome. These statistics suggest that measuring troponin is a highly sensitive-but not a highly specific — test in settings of a low pretest probability.

This case review will discuss the differential diagnoses of elevated cardiac troponin when acute coronary syndrome has been excluded.

1. Demand ischemia refers to a mismatch between myocardial oxygen demand and supply. Myocardial oxygen demand increases in the setting of tachycardia, increased afterload, systemic inflammatory response syndrome (SIRS), sepsis, and septic shock. Decreased oxygen delivery can occur in tachycardia, hypotension, and hypovolemia. Each of these conditions is either 
caused by, or leads to, tachycardia, a situation in which myocardial oxygen demand increases and supply decreases. In tachycardia the myocardium requires more oxygen to maintain its increased contractile rate; however, diastolic time also shortens, which has a net effect of limiting blood flow to the coronary arteries, thereby limiting oxygen supply. In the case of hypovolemia or hypotension, there is reduced perfusion pressure during systole, also leading to decreased oxygen supply to the myocardium. This set of circumstances can result in the release of cardiac troponin into circulation.

2. Sepsis is another clinical situation in which physicians often detect elevations of cardiac troponin. Amman et al studied 20 septic ICU patients, 17 of whom were noted to have elevated troponin levels. Troponin measurements ranged from 0.17 to 15.4. Ten of these patients had no history of coronary artery disease (CAD). Of the five patients who subsequently expired, eighty percent had no demonstrable CAD on post mortem. This study suggests that elevations of cardiac troponin in the setting of sepsis is not uncommon, and may occur in patients who otherwise show no evidence of CAD. The mechanism of troponin release in the setting of sepsis has not been completely elucidated. Endotoxin release from gram-negative organisms has been shown to lead to ventricular dilatation and myocardial depression. However, in Amman's study the majority of patients had gram-positive sepsis. It has been postulated that troponin release in sepsis may be due to increased membrane permeability. One possibility is that cytokines and interleukins released during sepsis cause the degradation of troponin to smaller, low molecular weight forms which are release through a more permeable membrane.

3. Left ventricular hypertrophy is also on the differential for elevated troponin. Hamwi et al measured troponin levels in 207 consecutive patients referred for echocardiogram. After excluding 124 on the basis of acute coronary syndrome and 29 on the basis of suboptimal echocardiogram, Hamwi's group determined that 8 of 74 patients had troponins out of the normal range. These 8 patients had a significantly higher mean left ventricular mass as compared to those with normal troponin levels. One hypothesis proposed in the article suggested that increased muscle mass would invariably result in increased oxygen demands. Furthermore, the increased muscle mass would potentially lead to decreased flow reserve secondary to "remodeled coronary microcirculation." Although intriguing, this article represents, at best, a correlation between enlarged muscle mass and troponin elevation, and is far from a rigorous proof of causality. Indeed, as Hamwi's group itself concludes, " $[\mathrm{w}]$ hether patients with combined troponin-I elevation and LV hypertrophy represent a subgroup with a higher risk of adverse cardiovascular outcome needs to be further determined."
4. Myocardial strain results when the myocardium is subjected to volume or pressure overload as in the case of congestive heart failure, damage to myofibrils may occur. A correlation has been demonstrated between elevated troponin and BNP levels in this setting as well as between myocardial stretch and apoptosis. Increased wall stress may also lead to decreased subendothelial perfusion, resulting in troponin release and decreased left ventricular function. Elevation of troponin tends to be associated with worse outcomes. In chronic heart failure, activation the sympathetic nervous system and inflammatory cytokines by the renin-angiotensin system may lead to myocyte injury and death, thus explaining elevated troponin levels in this setting. It is worth noting that troponin elevation is evident in up to $50 \%$ of people with a diagnosis of acute pulmonary embolism. Although not useful for diagnosis, the presence of an elevated troponin level in the setting of pulmonary embolism does portend a worse prognosis, as it suggests a significant clot burden against which the heart is unable to pump.

5. Other causes include vasospasm, trauma, pericarditis, myocarditis and renal failure. Vasospasm, or Prinzmetal's angina, can produce prolonged periods of ischemia, leading to the release of troponin in the absence of myocardial necrosis. Similarly, vasospasm has been offered as a possible explanation for elevated troponin in normal subjects following extended periods of exercise. Troponin rise has also been documented in cases of ischemic strokes and subarachnoid hemorrhage. Additionally, troponin is released in a significant number of people who incur blunt thoracic trauma. Hasdamir et al measured cardiac troponins in 35 patients who were hospitalized following ICD shocks. They demonstrated that troponin levels are elevated in the majority of patients who receive multiple shocks from their ICD, but only a small percentage of the elevations are due to an acute coronary syndrome. Elevation of troponin can also be seen in acute pericarditis and myocarditis as well as chronic renal failure.

\section{Conclusion}

In this patient none of the aforementioned causes could sufficiently explain her persistent elevation of troponin. It is clear, though, that given the multiple potential causes of troponin elevations outside of acute coronary syndrome, an individual's clinical presentation needs to considered thoroughly before patients are subjected to expensive—and potentially risky—diagnostic studies.

\section{References}

Jeremias A, Gibson MC. Narrative review: alternative causes for elevated cardiac troponin levels when acute coronary syndromes are excluded. Annals of Internal Medicine 2005; 142: 786-791.

Bakshi TK, Choo MK, Edwards CC et al. Causes of elevated troponin I with a normal coronary angiogram. Internal Medicine Journal 2002; 32:520.

Ammann P, Fehr T, Minder EI et al. Elevation of troponin I in sepsis and septic shock. Intensive Care Medicine 2001; 27: 965-969.

Hamwi, SM, Sharma, AK, Weissman, NJ et al. Troponin-I elevation in patients with increased left ventricular mass. American Journal of Cardiology 2003; 92:88-90.

Ng SM, Krishnaswamy P, Morrisey R, et al. Mitigation of the clinical significance of spurious elevations of cardiac troponin I in settings of coronary ischemia using serial testing of multiple cardiac markers. American Journal of Cardiology 2001; 87:994-999. 